\title{
Myelom: Lenalidomid-Doublette oder Triplet?
}

\author{
Die Kombination Lenalidomid/Dexamethason kann bei älteren Patienten \\ mit multiplem Myelom (MM) die Prognose verbessern. In einer Studie wurde \\ getestet, ob alkylierende Substanzen sich zusätzlich günstig auswirken.
}

tandardregime zur Behandlung von SMM-Patienten, die $>65$ Jahre alt sind oder für die eine autologe Stammzelltransplantation (ASCT) nicht infrage kommt, sind Melphalan-Prednison-Thalidomid (MPT) und Melphalan-Prednison-Bortezomib (VMP). Doch Lenalidomid-haltige Regimes sind diesen älteren Regimes überlegen. Um zu überprüfen, ob Alkylanzien einen zusätzlichen Nutzen bringen, wurden in einer Phase-IIIStudie 2 Lenalidomid-haltige Triplet-Regimes (Melphalan-Prednison-Lenalidomid [MPR] oder CyclophosphamidPrednison-Lenalidomid [CPR]) mit der Kombination Lenalidomid/niedrigdosiertes Dexamethason (Rd) verglichen. Untersucht wurden 662 Patienten mit neu diagnostiziertem MM, bei denen eine ASCT nicht infrage kam. Die Pati- enten wurden auf eine Induktionstherapie mit 9 28-tägigen Zyklen MPR $(\mathrm{n}=217)$, CPR $(\mathrm{n}=220)$ oder $\mathrm{Rd}(\mathrm{n}=217)$ randomisiert. Die MPR-Patienten erhielten $10 \mathrm{mg}$ Lenalidomid pro Tag über 21 Tage, orales Melphalan 0,18 mg/kg (Altersgruppe: 65-75 Jahre) oder 0,13 mg/kg (> 75 Jahre) über 4 Tage und 1,5 mg/kg Prednison über 4 Tage. Die CPR-Patienten bekamen neben Lenalidomid orales Cyclophosphamid über 28 (65-75 Jahre) oder 21 Tage ( $>75$ Jahre) und Prednison. Die Rd-Patienten erhielten 25 mg Lenalidomid pro Tag über 21 Tage und an den Tagen 1, 8, 15 und $2240 \mathrm{mg}$ (65-75 Jahre) oder $20 \mathrm{mg}$ (> 75 Jahre) Dexamethason. Primärer Endpunkt war das progressionsfreie Überleben (PFS) unter den Triplet-Regimes (MPR und CPR) versus Doublet-Regime (Rd).
Nach medianem Follow-up von 39 Monaten betrug das mediane PFS 22 Monate in den Triplet-Armen und 21 Monate im Doublet-Arm ( $\mathrm{p}=0,284)$. Das mediane Gesamtüberleben (OS) wurde in keinem Arm erreicht. Die Rate für das 4-Jahres-OS betrug $67 \%$ in den Triplet-Armen und $58 \%$ im Doublet-Arm $(\mathrm{p}=0,709)$. Auch wenn die 3 Arme einzeln analysiert wurden, zeigten sich keine Unterschiede hinsichtlich des Therapieerfolgs. Die häufigste Nebenwirkung vom Grad $\geq 3$ war Neutropenie (MPR: 64\%, CPR: $29 \%$ und Rd: $25 \%$; $<<0,0001)$. Nicht-hämatologische Nebenwirkungen vom Grad $\geq 3$ waren in allen Armen ähnlich häufig.

Fazit: In der Studie waren Lenalidomidbasierte Triplet-Regimes, die ein Alkylans enthielten, bei der Therapie älterer MM-Patienten der Kombination Rd nicht überlegen. Letztere war verträglicher.

Kathrin von Kieseritzky

Magarotto V et al. Triplet vs doublet lenalidomide-containing regimens for the treatment of elderly patients with newly diagnosed multiple myeloma. Blood. 2016;127(9):1102-8.

\section{MM: Lenalidomid verträglicher als Thalidomid?}

\author{
Ist für ältere Patienten mit multiplem Myelom (MM) die Therapie mit \\ Lenalidomid/Melphalan/Prednison verträglicher als die mit Thalidomid/ \\ Melphalan/Prednison?
}

D ie Kombination von Melphalan, Prednison und Thalidomid (MPT) ist derzeitig eine Standardtherapie für ältere Patienten mit neu diagnostiziertem MM, für die eine Stammzelltransplantation nicht infrage kommt.

Die Langzeitbehandlung mit Thalidomid birgt eine erhöhte Gefahr für Neurotoxizität, weshalb ein Austausch dieser Substanz gegen das ebenfalls wirksame Lenalidomid infrage kommen könnte. In der multizentrischen randomisierten Studie wurden 668 Patienten, die entweder $>65$ Jahre alt waren oder jünger, aber aufgrund ihrer Verfassung nicht für eine Hochdosistherapie und Stammzelltransplantation infrage kamen, in zwei Therapiearme randomisiert.

318 Patienten erhielten 9 4-wöchige Zyklen MPT, gefolgt von einer Thalido-
mid-Erhaltungstherapie (100 mg täglich) bis zum Krankheitsprogress oder Auftreten unakzeptabler Nebenwirkungen (MPT-T). Bei 319 Patienten wurde Thalidomid durch Lenalidomid ersetzt, und sie erhielten 9 vierwöchige Zyklen MPR, gefolgt von einer Lenalidomid-Erhaltungstherapie (10 mg an den Tagen 1-21 eines 28-tägigen Zyklus; MPR-R) bis zum Krankheitsprogress.

Primärer Endpunkt war das progressionsfreie Überleben (PFS). Nach medianem Follow-Up von 36 Monaten betrug das PFS unter MPT-T 20 Monate (95\%-Konfidenzintervall [95\%-KI] 1823 Monate) und unter MPR-R 23 Monate (95\%-KI 19-27 Monate; Hazard Ratio 0,87, $95 \%$-KI 0,72-1,04; $\mathrm{p}=0,12$ ). Die Gesamtansprechraten waren in beiden Therapiearmen mit $81 \%$ unter MPT-T und $84 \%$ unter MPR-R ähnlich hoch. Komplettremissionen zeigten sich bei $10 \%$ der Patienten unter MPT-T und $13 \%$ unter MPR-R.

Hämatologische Nebenwirkungen traten unter MPR-R häufiger auf, insbesondere Grad-3/4-Neutropenie (64\% unter MPR-R vs. $27 \%$ unter MPT-T). Neuropathien vom Grad $\geq 3$ waren im MPT-T-Arm signifikant häufiger (16 vs. $2 \%$ ), was zu einer deutlich geringeren Dauer der Erhaltungstherapie mit Thalidomid (5 vs. 17 Monate) führte - unabhängig vom Lebensalter der Patienten.

Fazit: Hinsichtlich der Effizienz unterschieden sich die beiden Regimes MPTT bzw. MPR-R nicht. Unter MPT-T kam es häufiger zu schwerer Neuropathie, unter MPR-R stand die Myelosuppression als Nebenwirkung im Vordergrund.

Kathrin von Kieseritzky

Zweegman S et al. Melphalan, prednisone, and lenalidomide versus melphalan, prednisone, and thalidomide in untreated multiple myeloma. Blood. 2016;127(9):1109-16. 